\title{
Laboreal
}

Volume 15 N$^{\circ} 2$ | 2019

Varia

\section{Jacques Leplat y la psicología ergonómica}

Jacques Leplat e a psicología ergonómica

Jacques Leplat et la psychologie ergonomique

Jacques Leplat and ergonomic psychology

\section{Régis Ouvrier-Bonnaz}

Traductor. Francisca Baldrich Advis

\section{OpenEdition}

\section{Journals}

Edición electrónica

URL: http://journals.openedition.org/laboreal/14912

DOI: 10.4000/laboreal. 14912

ISSN: 1646-5237

Editor

Universidade do Porto

\section{Referencia electrónica}

Régis Ouvrier-Bonnaz, « Jacques Leplat y la psicología ergonómica », Laboreal [En línea], Volume 15 No2 | 2019, Publicado el 01 diciembre 2019, consultado el 24 septiembre 2020. URL : http:// journals.openedition.org/laboreal/14912 ; DOI : https://doi.org/10.4000/laboreal.14912

Este documento fue generado automáticamente el 24 septiembre 2020.

\section{cc) (i) (8)}

Laboreal está licenciado com uma Licença Creative Commons - Atribuição-NãoComercial 4.0 Internacional. 


\title{
Jacques Leplat y la psicología ergonómica
}

\author{
Jacques Leplat e a psicología ergonómica \\ Jacques Leplat et la psychologie ergonomique \\ Jacques Leplat and ergonomic psychology
}

\section{Régis Ouvrier-Bonnaz}

Tradución : Francisca Baldrich Advis

El 17 de octubre de 2019, Jacques Leplat cumplió 98 años.

2 En 1949, empujó por primera vez la pesada puerta del Instituto nacional de estudios del trabajo y de la orientación profesional (INETOP) ubicado en el 41 de la calle Gay Lussac en el 5to distrito de Paris para seguir durante dos años la formación de consejero de orientación escolar y profesional. Setenta años más tarde, el viernes 19 de abril de 2019, en este sitio histórico del desarrollo de la psicología del trabajo, de la orientación y de la ergonomía [1], una jornada, titulada "Con Jacques Leplat en el 41 de Gay Lussac », es organizada por los equipos de ergonomía, de psicología del trabajo y de clínica de la actividad a iniciativa del Grupo de investigación y de estudio sobre la historia del trabajo y de la orientación (GRESTHO) al que se unió desde su creación en el Centro de investigación sobre el trabajo y el desarrollo (CRTD) del Conservatorio nacional de artes y oficios.

3 Todos aquellos que frecuentan o que frecuentaron el 41 de la rue Gay Lussac cruzaron su delgada silueta subiendo sin dificultad, a paso enérgico, las escaleras que llevan a los cinco pisos del edificio para llegar a su oficina o a la de sus colegas. Apasionado por las carreras en montaña, en particular en los Alpes, que descubrió joven y que continuó recorriendo durante su tiempo libre, el esfuerzo físico y el esfuerzo intelectual seguirán siendo para él, como nos lo dirá muchas veces, une necesidad vital. Muchos recuerdan sus amables visitas a sus oficinas y su eterna pregunta : ¿ ¿sobre qué trabaja usted en este momento?». Los intercambios con las demás personas en su disciplina, la ergonomía, pero asimismo en las disciplinas vecinas, le interesan sobremanera. Durante una entrevista con la SELF en 2002, precisando que el “ problema práctico » es 
siempre multidisciplinario, no dudaría en citar a Marc Bloch (1886-1944), el historiador fundador de los Anales de historia económica y social en 1929 : “ La ciencia descompone la realidad con la finalidad de observarla mejor, gracias a un juego de fuegos cruzados cuyos rayos se combinan y se interpretan constantemente. El peligro empieza cuando cada proyector pretende ver todo por él mismo, cuando cada cantón del conocimiento de toma por una patria " [2].

Titular del diploma de estado de consejero de orientación escolar y profesional y de la licencia de psicología recién creada preparada en paralelo, se unió en 1951 al Centro de Estudios y de Investigación Psicotécnica (CERP) financiado por el Ministerio del Trabajo bajo el consejo de Paul Fraisse (1911-1996) entonces profesor de psicología de la Universidad de Paris, que dirigirá su tesis "La noción de aplicación en psicología del trabajo ", defendida en 1974. Permanecerá quince años en este establecimiento público cuya primera misión es de gestionar la concepción y la organización de exámenes psicológicos previos a la entrada de los alumnos en práctica en los centros de formación profesional de la Asociación para la formación profesional de adultos (AFPA), sucediendo en 1959 a Jean-Marie Faverge (1912-1988) en la dirección.

Desde su llegada al CERP, entonces dirigida por André Ombredane (1898-1958), desarrolló y adaptó pruebas y cuestionarios para un público identificado como discapacitado, dos artículos publicados en el Boletín del CERP dan cuenta de sus primeros trabajos [3]. Rápidamente fue asociado al servicio de Faverge que acababa de llevar a cabo una misión de observación y de estudio de los laboratorios de psicología aplicada al trabajo en los Estados Unidos, financiada por el Comisariado General para la productividad, una especie de Comisión de Planificación. Faverge [4] descubre ahí los laboratorios de "Human engineering" y describe en su reporte un mundo donde domina el equipamiento técnico, desconocido en Francia [5]. Asimismo descubre, durante este viaje, el libro de Chapanis y de sus colaboradores "Applied Experimental Psychology " [6], sobre el cual le solicita a Jacques Leplat hacer un informe. Éste redacta entonces para el boletín del CERP creado en 1952 lo que podríamos considerar como su primer artículo científico "Adaptation de la machine à l'homme " "Adaptación de la máquina al hombre »). Como señala en una entrevista realizada para la revista Pistes en 2005['], este articulo marca el comienzo de una nueva orientación del CERP, que desde aquel momento, se interesa en los métodos de análisis del trabajo que sus investigadores no cesarán de estudiar y desarrollar [8]. Es dicho artículo el cual la revista Laboreal ahora publica junto con una bibliografía selecta de Jacques Leplat, agrupando la mayor parte de sus escritos, compilada por la Biblioteca y el Centro de documentación sobre la formación y el trabajo (CDFT) del CNAM ubicado en la calle Gay Lussac. Dada la gran cantidad de reseñas de libros, esto último confirma, si fuera necesario, la atención que siempre ha prestado al trabajo de los demás. Sus presentaciones, a su vez, suscitaron siempre el interés de los investigadores de la comunidad pero también de los profesionales de su ámbito. Es así como Yves Clot, concluyendo la jornada de homenaje, señala haberse sentido alentado a seguir la carrera universitaria que estaba considerando, luego de leer el reporte de Jacques Leplat en el Trabajo humano, de un libro cuyo prefacio él habría redactado en 1981 [ ${ }^{9}$. $\mathrm{Su}$ obra, en el sentido en que Ignace Meyerson lo entiende (1948) [ ${ }^{10}$ ], es un valioso caleidoscopio para escudriñar el espesor de los territorios de la ergonomía y de la psicología del trabajo hoy en día. 
6 En el artículo presentado aquí, luego de haber definido lo que cubre la "Human engineering " y de haber adoptado una posible traducción al francés : “ adaptation de la machine à l'homme » (" adaptación de la máquina al hombre »), define una clasificación para exponer dos tipos de investigación que juzga características de lo que se hace en este ámbito: el estudio de los dispositivos de control y el estudio de los mandos. Concluye : "la introducción de la experimentación en el estudio del trabajo, un terreno en el cuál el empirismo ha sido durante mucho tiempo la única regla, permitirá indudablemente alcanzar importantes progresos tanto en el nivel puramente técnico (mejora del material) y pedagógico (aprendizaje más racional de la tarea) como en el nivel más estrictamente psicológico (orientación y selección)» (1953, p. 38). Por ende lo que interesa a Jacques Leplat es la relación entre técnica y psicología que permite pensar el estudio del sistema hombre/ máquina como una unidad, como un todo. Este interés es clave para aprehender mejor la orientación dada a gran parte de sus estudios e investigaciones.

7 En un artículo publicado el mismo año en L'Année psychologique (El año psicológico) titulado "Trabajos recientes de "tecnología humana" (Human engineering)» $\left[{ }^{11}\right]$, que realiza una amplia revisión de los temas como lo atestigua la bibliografía, esboza un primer análisis para comprender mejor las posibles relaciones entre una psicología experimental fundamental y una psicología experimental aplicada. Como afirma en su tesis, "si es bueno, en efecto, distinguir un objetivo fundamental y un objetivo de aplicación, esta distinción no debería conducir a aislar los pasos que se inscriben en estos dos objetivos (...) la psicología del trabajo fundamental y aplicada deben estar articuladas de manera estrecha $y$ coherente (1974, p. 158). Por consiguiente, no dejará de estudiar la relación dialéctica existente entre estas dos orientaciones de la psicología, una que proporciona modelos de inteligibilidad, y la otra, soluciones concretas susceptibles de ayudar a mujeres y hombres a enfrentar las tareas que tienen que llevar a cabo en sus trabajos. De este enfoque deriva la definición del campo específico a la psicología ergonómica que la diferencia de la psicología cognitiva : estudio clínico de la interacción del hombre con su entorno laboral, con el fin de actuar sobre los dispositivos socio-técnicos para mejorar las condiciones de vida de mujeres y hombres en el trabajo $\left.{ }^{12}\right]$.

\section{NOTAS}

1. Ver sobre este tema Régis Ouvrier-Bonnaz, «A psicologia em França de 1870 a 1940, de uma ciência aplicada a uma disciplina universitária », Laboreal [En línea], Volume 3 №1 | 2007, Publicado el 01 julio 2007, consultado el 21 noviembre 2019. URL : http:// journals.openedition.org/laboreal/13016

2. Marc Bloch (1993). Apologie pour l'histoire ou métier d'historien. Paris : Armand Colin.

3. J. Leplat (1953). L'examen psychotechnique des aveugles. Bulletin du CERP, II, 2-3, 23-39 et (1954). Étude d'une batterie de tests pour aveugles. Bulletin du CERP, III, 1, 9-15.

4. Sobre Ombredane y Faverge en el CERP, ver R. Ouvrier-Bonnaz y A. Weill-Fassina. André Ombredane (1898-1958) et Jean-Marie Faverge (1912-1988). L'analyse du travail, ruptures et évolutions. Toulouse : éditions Octares (versión electrónica en acceso libre - https:// www.octares.com) 
5. Citado por J. Leplat (2002) en una entrevista realizada en la comisión de historia de la SELF. (https://ergonomie-self.org).

6. Chapanis, A., Gardner, W.-R., Morgan, C.-T. (1949). Applied experimental psychology. NewYork : John Wiley.

7. Esther Cloutier et Corinne Gaudart, «Entrevue guidée avec Jacques Leplat ", Perspectives interdisciplinaires sur le travail et la santé [En ligne], 7-1 | 2005, mis en ligne le 01 février 2005, consulté le 21 novembre 2019. URL : http://journals.openedition.org/pistes/3250 ; DOI : 10.4000/ pistes. 3250

8. Estos estudios, llevados a cabo en la industria textil, la industria de máquinas-herramientas y con fabricantes de artefactos para obras de construcción, son presentados y discutidos en J.-M. Faverge, J. Leplat et B. Guiguet (1958). L'adaptation de la machine à l'homme ». Paris : PUF.

9. Se trata de la obra de Y. Oddone, A. Alessandra et G. Briante (1981). Redécouvrir l'expérience ouvrière. Vers une autre psychologie du travail ? Paris : Éditions Sociales.

10. I. Meyerson (1948). Les fonctions psychologiques et les œuvres. Paris : Librairie philosophique J. Vrin. Para este autor: « La acción, el pensamiento humano, se expresan mediante las obras (...). La mente nunca se expresa en el vacío; se le conoce sólo en el trabajo, en sus manifestaciones dirigidas, expresadas, conservadas » (p. 10).

11. J. Leplat (1953). «Travaux récents de "technologie humaine" (Human engineering)». L’Année psychologique, LIII, 2, 517-537.

12. Ver J. Leplat (1980). La psychologie ergonomique. Paris : PUF.

\section{AUTORES}

\section{RÉGIS OUVRIER-BONNAZ}

Groupe de Recherches sur l'Histoire du Travail et de l'Orientation (GRESHTO)

Centre d'Études sur le travail et le développement (CRTD)

Centre National des Arts et Métiers (CNAM), 41 Rue Gay-Lussac, 75020 Paris, France

regis.ouvrier_bonnaz@cnam.fr 